\title{
Radiation Dose for 345 CT-Guided Interlaminar Lumbar Epidural Steroid Injections
}

\author{
A.L. Chang, A.H. Schoenfeld, A.L. Brook, and T.S. Miller
}

\begin{abstract}
BACKGROUND AND PURPOSE: CT guidance is increasingly being used to localize the epidural space during epidural steroid injections. A common concern is that CT may be associated with significantly higher radiation doses compared with conventional fluoroscopy. The goal of this retrospective study was to determine the average dose-length product and effective dose delivered while interlaminar epidural steroid injections are performed and allow comparison with other modalities.
\end{abstract}

MATERIALS AND METHODS: A total of 281 patients who had undergone 345 consecutive CT-guided epidural steroid injections of the lumbar spine were evaluated for radiation exposure. The dose-length product for each scan was derived from the CT dose index volume and scan length. Effective dose was then calculated from the dose-length product and a $\kappa$ coefficient of 0.015 . Procedure time was calculated from the PACS time stamp on the scout image to the last CT image of the last image series.

RESULTS: The average dose-length product across all procedures was $89.6 \pm 3.33 \mathrm{mGy} \cdot \mathrm{cm}$, which represents an effective dose of $1.34 \pm$ $0.05 \mathrm{mSv}$. No complications from the procedure were observed, and average procedure time was 8 minutes.

CONCLUSIONS: The use of a stationary table and an intermittent scanning technique allow for short procedures and doses that are significantly lower than those of conventional diagnostic CT scans. Furthermore, because CT dose index overestimates radiation dose in stationary table procedures, the actual radiation dose may be even lower than stated here.

ABBREVIATIONS: $\mathrm{CTDI}=\mathrm{CT}$ dose index $\mathrm{CTDI}_{\mathrm{vol}}=\mathrm{CTDI}$ volume; $\mathrm{DLP}=$ dose-length product; $\mathrm{ED}=$ effective dose; $\mathrm{ESI}=$ epidural steroid injection

C guidance has been increasingly adopted for use in interventional procedures of the spine, including epidural steroid injections (ESIs). ESIs are performed by injection of a corticosteroid solution, often with a local anesthetic, into the epidural space of the spine. This procedure is frequently used to treat radicular pain from herniated disks and spinal stenosis. CT offers several advantages versus traditional fluoroscopy, including the ability to visualize the soft tissues and provide higher anatomic precision and more accurate needle placements, and the option of using air instead of contrast medium to localize the epidural space. ${ }^{1}$ Accurate localization of the epidural space and needle position during ESIs is crucial, as it facilitates precise delivery of medications and reduces the risks for needle misplacement and subsequent complications. Previous reports on blind injections have shown incor-

Received November 11, 2012; accepted after revision January 22, 2013.

From the Department of Radiology (A.L.C., A.H.S., A.L.B., T.S.M.), Montefiore Medical Center, Albert Einstein College of Medicine, Bronx, New York; and Department of Radiological Sciences (A.L.C.), University of California, Irvine, California.

Please address correspondence to Todd Miller, MD, Montefiore Medical Center Department of Radiology, 111 East 210th St, Bronx, New York, 10467; e-mail: tmiller@montefiore.org

http://dx.doi.org/10.3174/ajnr.A3540 rect placement in up to $25 \%$ of cases, even when performed by experienced providers. ${ }^{2}$ We recently reported our experience of safely performing 1000 procedures with CT guidance and air contrast to localize the epidural space. ${ }^{1}$ The goal of this study was to analyze a cohort of similar patients to evaluate the dose-length product (DLP) and effective dose (ED) delivered while interlaminar ESI was being performed.

\section{Review of CT Dose Index, Dose-Length Product, and Effective Dose}

CT dose index (CTDI) is a commonly used measure of dose in CT dosimetry. It is defined by the formula

$$
C T D I=\frac{1}{N T} \int_{-\propto}^{\propto} D(z) d z,
$$

where $N$ is the number of sections in a single axial scan, $T$ is equivalent to the width of sections imaged by 1 channel of a multidetector row $\mathrm{CT}$, and $D(z)$ represents the radiation dose profile along the $\mathrm{z}$-axis of the scanner. CTDI is an estimate of the average dose to a central volume receiving contiguous scans along the $\mathrm{z}$-axis 
and includes the contribution to the dose from overlap of dose tails. ${ }^{3,4}$ The measurement of CTDI is made with a pencil-shaped ionization chamber with a standard length of $100 \mathrm{~mm}$ in an acrylic cylindrical phantom, which gives rise to the definition of

$$
C T D I_{100}=\frac{1}{N T} \int_{-50 \mathrm{~mm}}^{50 \mathrm{~mm}} D(z) d z .
$$

Within the cylindrical phantom, the dose is higher at the periphery of a given section compared with the center, and a weighted version of $C T D I_{100}$ is defined as

$$
C T D I_{W}=\frac{1}{3} C T D I_{100 \text { center }}+\frac{2}{3} C T D I_{100 \text { edge }}
$$

which averages the dose over the field of view. A 16-cm diameter cylinder is used to measure $\mathrm{CTDI}_{w}$ for head examinations, whereas a diameter of $32 \mathrm{~cm}$ is used for body examinations.

The most commonly used form of CTDI in dosimetry is $\mathrm{CTDI}_{\text {vol }}$, which also incorporates the scanner's pitch into the dose estimate:

$$
C T D I_{v o l}=\frac{1}{\text { pitch }} C T D I_{W}
$$

where

$$
\text { pitch }=\frac{1}{N T}
$$

and $I$ represents the table increment in millimeters $(\mathrm{mm})$ per axial scan. CTDI $_{v o l}$ is easily calculated and is available in most scanner output dose tables. The SI unit of measurement of the dose is the milligray ( $\mathrm{mGy}$ ).

Because scans of greater physical length increase with the proportion of the body exposed to radiation and thus affect the radiation dose, the DLP was developed to incorporate scan length into the dose calculation: $D L P=C T D I_{v o l} \times$ scan length $(\mathrm{cm})$. The units of DLP are in milligray centimeters $(\mathrm{mGy}-\mathrm{cm})$ and represent the total energy absorbed in the scanned volume.

Lastly, one other common index in dosimetry is ED (measured in millisievert $[\mathrm{mSv}]$ ), which takes into account that a given radiation dose has different effects on tissues with different sensitivities to radiation. The ED is defined as the summation of the dose to a series of organs multiplied by their weighting factors. However, in CT examinations, the ED can be approximated by the simplified relationship, $E D=\kappa \times D L P$, where $\kappa$ is the conversion coefficient from DLP to ED. The value of the coefficient $\kappa$ depends on the region of the body being scanned. Effective dose is most useful when used as a parameter to compare relative exposure/risk between different types of imaging studies. ${ }^{5}$

\section{MATERIALS AND METHODS \\ Study Design}

After receiving approval from the institutional review board, we conducted a retrospective review on 281 consecutive patients undergoing 345 CT-guided interlaminar ESIs in the lumbar spine at a single institution during an 8 -month period. All procedures were performed by 1 of 3 interventional neuroradiologists who perform at least $500 \mathrm{CT}$-guided procedures annually. Patients were seen in an office consultation before the procedure, moni-

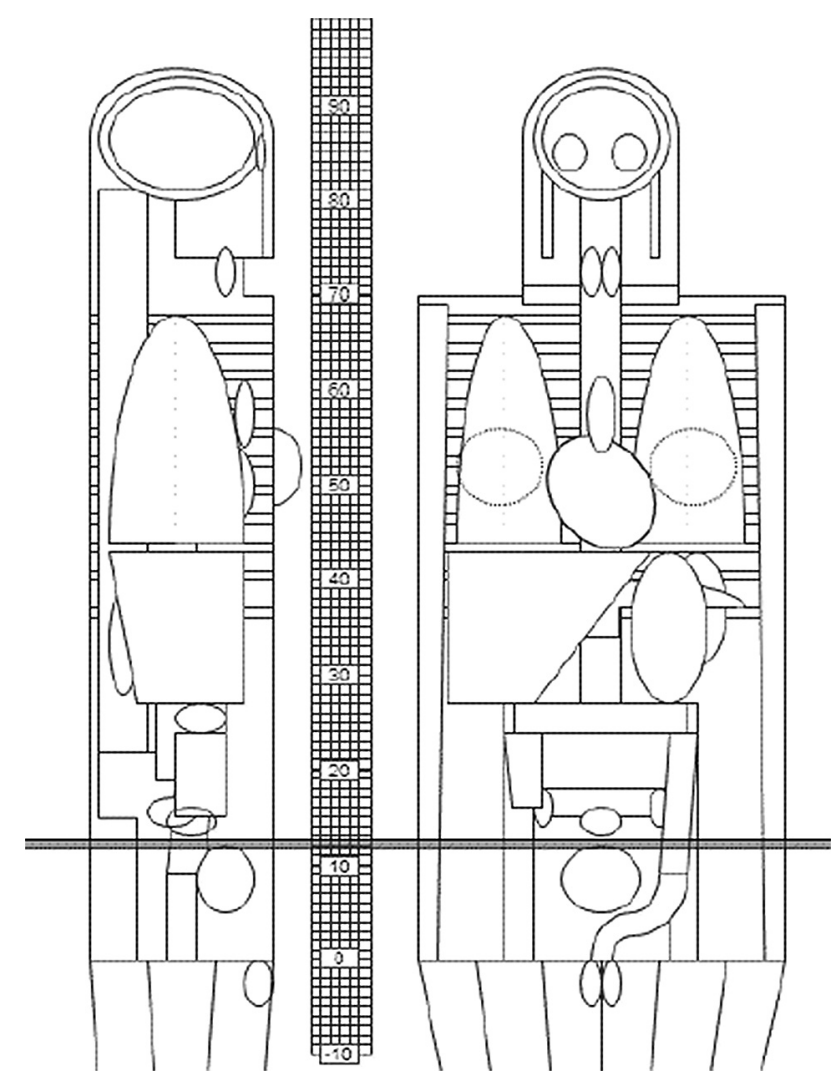

FIG 1. Approximate anatomic region used in ImPACT dosimetry software.

tored for 1 hour immediately after the procedure, contacted by phone at 24 hours, and seen in the office 1 month later.

There are several different modes possible with CT; one option is to use CT in a fluoroscopic mode, which provides continuous real-time image collection in a manner similar to traditional fluoroscopy. Another option is to use intermittent scans, where single images are taken serially, either with a foot pedal or remotely at the control station. These scans all occur on a stationary table, so that the same cross section is continually acquired. In our study, images were obtained by use of an intermittent scanning mode. The procedures were conducted at $120 \mathrm{kVp}$ and $50 \mathrm{~mA}$; in a few instances, the milliampere needed to be increased to provide better resolution in patients with a large body habitus.

$\mathrm{CTDI}_{v o l}$ and DLP displayed in the CT scanner dose report were recorded for each patient. The $\mathrm{CTDI}_{v o l}$ was also measured under standard conditions with acrylic phantoms and compared with the displayed value to verify its accuracy. The mean deviation of measured versus reported dose was $+1.9 \%$ (range, $-6 \%$ to $+12 \%$ across scanners). The images for each patient were individually reviewed to ensure that each procedure was correctly categorized. We obtained procedure time by comparing the scout image with the last CT image.

\section{Dose Calculations}

Once the DLP had been collected for each individual case, an ED/DLP conversion coefficient was calculated from the ImPACT CT Patient Dosimetry Calculator version 1.0.2 (ImPACT, London, United Kingdom), a widely available spreadsheet in the ra- 
List of all outliers at 2 SDs above the mean, and their causes

\begin{tabular}{cccl}
\hline Patient No. & DLP $(\mathbf{m G y} \cdot \mathbf{c m})$ & Scan Parameter & \multicolumn{1}{c}{ Cause } \\
\hline 1 & 826.91 & $120 \mathrm{kVp} 460 \mathrm{~mA}$ & Technical error, patient morbidly obese \\
2 & 551.78 & $120 \mathrm{kVp} 150 \mathrm{~mA}$ & Patient morbidly obese \\
3 & 362.39 & $120 \mathrm{kVp} 150 \mathrm{~mA}$ & Patient morbidly obese \\
4 & 319.39 & $120 \mathrm{kVp} 100 \mathrm{~mA}$ & Patient morbidly obese \\
5 & 306.79 & $120 \mathrm{kVp} 200 \mathrm{~mA}$ & Patient morbidly obese \\
6 & 257.26 & $120 \mathrm{kVp} 100 \mathrm{~mA}$ & Patient morbidly obese, long procedure time \\
7 & 216.66 & $120 \mathrm{kVp} 50 \mathrm{~mA}$ & Patient morbidly obese, technically difficult, \\
& & & long procedure time \\
\hline
\end{tabular}

diation dosimetry community, and by using ICRP 103 weighting factors (Fig 1). ${ }^{6}$ By taking into account the affected organs and their specific weighting factors, we estimated the ED/DLP coefficient to be approximately $15 \mu \mathrm{Sv} / \mathrm{mGy}^{\circ} \mathrm{cm}^{7,8}$

\section{Technique}

All procedures were performed with intermittent CT guidance by use of LightSpeed RT 16/LightSpeed Xtra scanners (GE Healthcare, Milwaukee, Wisconsin), with $120 \mathrm{kVp}, 50 \mathrm{~mA}$, and 0.8-second rotation time. Patients were placed in a prone position. The patient's back was then marked, prepped, and draped in the usual sterile manner. Several helical images through the target level were then performed to plan trajectory and identify an interruption between the laminae to allow the needle to reach the target epidural space from a posterior (dorsal interlaminar) approach. Lidocaine $2 \%$ was used to anesthetize the area, and a 22-gauge spinal needle was then advanced into the epidural space by use of an interlaminar approach. A 3-mL Luer-slip syringe with either 1 $\mathrm{mL}$ of air or contrast medium (iohexol $180 \mathrm{mg} / \mathrm{mL}$ ) was attached to the spinal needle, and a loss-of-resistance technique was used to guide the needle past the ligamentum flavum, and air or iohexol was injected to identify the epidural space. ${ }^{1}$ Additional axial images were then obtained to confirm correct placement of the needle. This was repeated until the epidural space was demonstrated with the injection. A steroid/anesthetic mixture of betamethasone sodium phosphate and betamethasone acetate $(12-18 \mathrm{mg})$ and bupivacaine hydrochloride $0.5 \%(1-3 \mathrm{~mL})$ was injected into the epidural space, and the needle was withdrawn ending the procedure. Any potential complications were recorded and reported according to Society of Interventional Radiology guidelines. ${ }^{9}$

\section{RESULTS}

A total of 281 patients underwent 345 CT-guided lumbar ESIs via an interlaminar approach. The average patient age was 59.7 years, 206 (59.7\%) were women, and 139 (40.2\%) were men. The mean DLP for all procedures was $89.6 \pm 3.33 \mathrm{mGy} \cdot \mathrm{cm}$, and the median value was $78.34 \mathrm{mGy}{ }^{*} \mathrm{~cm}$. By use of the above-stated ED/DLP conversion factor (also known as the coefficient) of $15 \mu \mathrm{Sv} /$ $\mathrm{mGy} \cdot \mathrm{cm}$, the obtained mean DLP converts to an estimated ED of $1.34 \pm 0.05 \mathrm{mSv}$. Average procedure time was $8.4 \pm 0.4$ minutes, beginning from scout image acquisition to the last axial image acquired. No complications were observed during the procedure or at 1-month office follow-up.

\section{DISCUSSION}

The use of CT as a guidance technique for minimally invasive procedures has brought many advantages and innovations.
The traditional drawback to CT use has been its association with increased risk from higher radiation exposures than fluoroscopy. ${ }^{10,11}$ There are several reasons to quantify radiation exposure in lumbar ESIs. Multiple ESIs are not uncommon for pain relief in some patients, some of whom present at a relatively young age. Repeated radiation exposure over the same anatomic area can increase both deterministic and stochastic radiation-related risks, which makes the determination of exposure from any diagnostic or therapeutic study important.

In our study, the average DLP from use of an interlaminar approach for ESIs was $89.6 \pm 3.33 \mathrm{mGy} \cdot \mathrm{cm}$ while using an intermittent scanning mode. The conversion of our average DLP to ED by an ED/DLP factor of $15 \mu \mathrm{Sv} / \mathrm{mGy} \cdot \mathrm{cm}$ yields a value of $1.34 \pm$ $0.05 \mathrm{mSv}$. By comparison, the average background radiation dose per person in the United States is approximately $3.11 \mathrm{mSv}$ per year, and a regular CT of the abdomen/pelvis is approximately 10 $\mathrm{mSv}{ }^{12}$ Intermittent scans also have the advantage of decreasing radiation exposure to the patient and operator, ${ }^{13}$ with the potential to completely eliminate exposure to the operator when image acquisition is initiated from within the shielded control station.

Our value compares favorably to lumbar radiographs in the lumbar spine, with an estimated $1-1.5 \mathrm{mSv}$ in studies done in the United Kingdom, by the NRCP, and by the UNSCEAR. ${ }^{12,14-16}$ Schmid et $\mathrm{al}^{17}$ conducted simulations by using Rando phantoms (The Phantom Library, Salem, New York) and estimated that the ED from 4-10 intermittent CT scans ranged from 1.51-3.53 mSv, with 4 scans estimated to be the average number necessary. Thus, our results are near the general predicted range. Schmid et al ${ }^{17}$ also found that 1-3 minutes of conventional fluoroscopy yielded an ED of 0.41-1.25 $\mathrm{mSv}$, respectively, with 1 minute being the estimated average fluoroscopy time necessary. Kim et al ${ }^{18}$ also performed a study using phantoms to estimate ESI dose with conventional fluoroscopy and found an estimated ED of $0.93 \mathrm{mSv}$ from a mean clinical procedure time of 40.7 seconds (range, 14.395.9 seconds).

Limited work has previously been done on the dose from CT fluoroscopy in ESI; however, Hoang et $\mathrm{al}^{19}$ recently compared CT fluoroscopy with conventional fluoroscopy in lumbar ESIs. They found that conventional fluoroscopy yielded $0.85 \mathrm{mSv}$ from a mean exposure time of 37 seconds, whereas CT fluoroscopy yielded $0.45 \mathrm{mSv}$ with exposure time of 4.7 seconds, though they also performed preliminary planning CTs, which added $2.90 \mathrm{mSv}$ to produce a total dose of $3.35 \mathrm{mSv}$ per procedure. ${ }^{19}$

A limitation of any study measuring radiation exposure involves the differences between studies in machine types, settings, and even operator skill, which can all affect the exposure time and dose. However, these studies do provide a useful context for understanding and comparing our results with known data on predicted doses for intermittent scans and conventional fluoroscopy.

A few values in our data were found to be causing a positive 


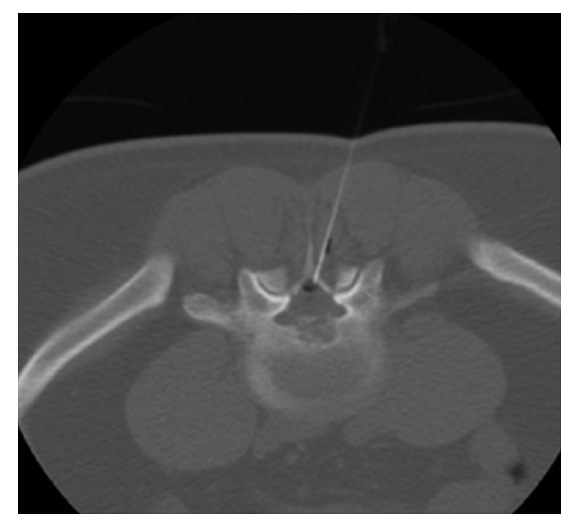

FIG 2. CT guidance used to localize the epidural space with adequate visualization by use of air contrast.

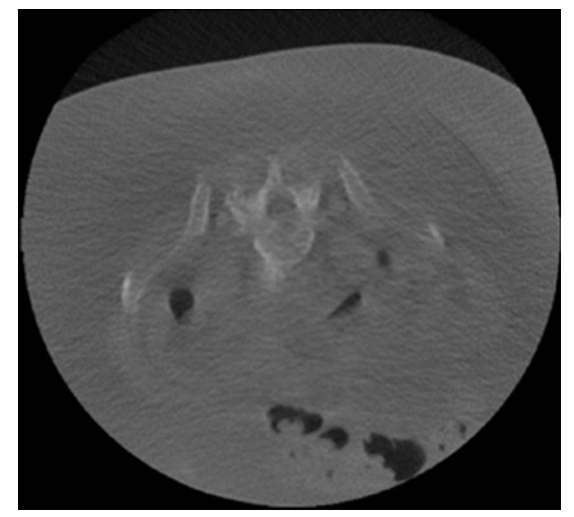

FIG 3. Limited resolution with $50 \mathrm{~mA}$ on a patient with a very large body habitus.

skew in our DLP; although the mean was $89.6 \mathrm{mGy}^{\circ} \mathrm{cm}$, the median value was $78.34 \mathrm{mGy}^{\circ} \mathrm{cm}$, and the DLP ranged from 11.67826.91. When evaluating all cases both above and below 2 SDs of the mean, we found 6 outliers, all of which were greater than the mean. After reviewing these cases individually (Table), we determined that in most cases of high DLP, the tube current had been increased to improve poor resolution in images because of excessive soft tissue mass in obese patients. In other cases, technically difficult studies contributed to long procedure times and greater CT usage. In a single case, the tube current had been incorrectly set to a higher value used in diagnostic imaging. Any of these factors can increase the received dosage.

We observed several limitations to our study. First, it is important to note that the CT machines used in our study did not use the currently available iterative reconstruction techniques that may reduce the dose by $40 \%-70 \%$ without loss of image quality. ${ }^{20}$

In addition, multiple factors could have caused overestimation of dose. High dose and time outliers typically occur in patients with a very large body habitus, where a higher milliampere is required to maintain adequate image resolution (Figs 2 and 3). ${ }^{21,22}$ This also underscores that ED data should not be used to estimate any given person's individual risk as age and body habitus significantly alter their radiation exposure, but rather as a parameter to compare exposures across different studies and procedures.
A factor that can cause an overestimation of ED stems from how CTDI is defined. The definition of CTDI assumes that the table is incrementally advanced through a scan length, which does not apply to interventional procedures utilizing a stationary table. CTDI includes the dose tails resulting from scatter in the integration of the dose, and these dose tails are reduced when a stationary table is used. Several groups have measured the peak skin dose to be only $50 \%-65 \%$ of the stated CTDI $100^{3,23,24}$ In fact, Leng et $\mathrm{al}^{23}$ used a correction factor of $0.6 \times \mathrm{CTDI}_{v o l}$ to correct for the dose in interventional intermittent stationary table scans. This finding implies that the intermittent CT dose is potentially even lower than the result reported here. Without very thin thermoluminescent or solid-state dosimeters, it is difficult to know how much lower the actual dose is than when estimated by the CTDI, and doing so would be a next logical step for further research.

The future of CT guidance for procedures in the spine is promising. The ability of CT to provide precise 3D needle localization combined with the radiation exposure results seen here, along with ongoing research currently being conducted on dose reduction methods for CT guidance, ${ }^{25}$ provides great future potential for the use of CT in spinal procedures.

\section{CONCLUSIONS}

The use of an intermittent scan mode can provide the advantages of CT guidance without necessarily subjecting the patient to excessive radiation; our study found an average ED of $1.34 \pm 0.05$ $\mathrm{mSv}$ per interlaminar ESI in the lumbar spine while using an intermittent scanning mode.

\section{REFERENCES}

1. Chang A, Pochert S, Romano C, et al. Safety of 1000 CT-guided steroid injections with air used to localize the epidural space. AJNR Am J Neuroradiol 2011;32:E175-177

2. White AH, Derby R, Wynne G. Epidural injections for the diagnosis and treatment of low-back pain. Spine 1980;5:78-86

3. Bauhs JA, Vrieze TJ, Primak AN, et al. CT dosimetry: comparison of measurement techniques and devices. Radiographics 2008;28:245-53

4. McCollough $\mathrm{CH}$, Leng S, Yu L, et al. CT dose index and patient dose: they are not the same thing. Radiology 2011;259:311-16

5. American Association of Physicists in Medicine. The Measurement, Reporting, and Management of Radiation Dose in CT. College Park, MD: American Association of Physicists in Medicine; 2008. AAPM Report No. 96

6. The 2007 Recommendations of the International Commission on Radiological Protection. ICRP publication 103. Ann ICRP 2007;37: $1-332$

7. Miller TS, Fruauff K, Farinhas J, et al. Lateral decubitus positioning for cervical nerve root block using CT image guidance minimizes effective radiation dose and procedural time. AJNR Am J Neuroradiol 2013;34:23-28

8. Huda W, Ogden KM, Khorasani MR. Converting dose-length product to effective dose at CT. Radiology 2008;248:995-1003

9. Sacks D, McClenny TE, Cardella JF, et al. Society of Interventional Radiology clinical practice guidelines. J Vasc Interv Radiol 2003;14:S199-S202

10. Brenner DJ, Hall EJ. Computed tomography-an increasing source of radiation exposure. $N$ Engl J Med 2007;357:2277-84

11. Mahesh M. Fluoroscopy: patient radiation exposure issues. Radiographics 2001;21:1033-45

12. National Council on Radiation Protection and Measurements. Report No. 160-Ionizing Radiation Exposure of the Population of the

AJNR Am J Neuroradiol 34:1882-86 Oct 2013 www.ajnr.org

1885 
United States (2009). Bethesda, MD: National Council on Radiation Protection and Measurements; 2009

13. Paulson EK, Sheafor DH, Enterline DS, et al. CT fluoroscopy-guided interventional procedures: techniques and radiation dose to radiologists. Radiology 2001;220:161-67

14. Alexander MD, Oliff MC, Olorunsola OG, et al. Patient radiation exposure during diagnostic and therapeutic interventional neuroradiology procedures. J Neurointerv Surg 2010;2:6-10

15. Hart D, Wall BF. Radiation Exposure of the UK Population from Medical and Dental X-Ray Examinations. Chilton, UK: National Radiological Protection Board; 2002. Publication no. NRPB-W4

16. Simpson AK, Whang PG, Jonisch A, et al. The radiation exposure associated with cervical and lumbar spine radiographs. J Spinal Disord Tech 2008;21:409-12

17. Schmid G, Schmitz A, Borchardt D, et al. Effective dose of CT- and fluoroscopy-guided perineural/epidural injections of the lumbar spine: a comparative study. Cardiovasc Intervent Radiol 2006;29:84-91

18. Kim S, Toncheva G, Anderson-Evans C, et al. Kerma area product method for effective dose estimation during lumbar epidural steroid injection procedures: phantom study. AJR Am J Roentgenol 2009;192:1726-30
19. Hoang JK, Yoshizumi TT, Toncheva G, et al. Radiation dose exposure for lumbar spine epidural steroid injections: a comparison of conventional fluoroscopy data and CT fluoroscopy techniques. $A J R$ Am J Roentgenol 2011;197:778-82

20. Schindera ST, Diedrichsen L, Müller HC, et al. Iterative reconstruction algorithm for abdominal multidetector $\mathrm{CT}$ at different tube voltages: assessment of diagnostic accuracy, image quality, and radiation dose in a phantom study. Radiology 2011;260:454-62

21. Uppot RN, Sahani DV, Hahn PF, et al. Effect of obesity on image quality: fifteen-year longitudinal study for evaluation of dictated radiology reports. Radiology 2006;240:435-39

22. Uppot RN, Sahani DV, Hahn PF, et al. Impact of obesity on medical imaging and image-guided intervention. AJR Am J Roentgenol 2007;188:433-40

23. Leng S, Christner JA, Carlson SK, et al. Radiation dose levels for interventional CT procedures. AJR Am J Roentgenol 2011;197:W97-103

24. Leng SV, Vrieze TJ, Lu Y, et al. A direct skin dose calculation method in CT scans without table motion: influence of patient size and beam collimation. Med Phys 2010;37:3110

25. Shepherd TM, Hess CP, Chin CT, et al. Reducing patient radiation dose during CT-guided procedures: demonstration in spinal injections for pain. AJNR Am J Neuroradiol 2011;32:1776-82 